Katharina Hamann

(Bergische Universität Wuppertal)

(D) https://orcid.org/0000-0001-9662-3322

Nadja Wulff

(Pädagogische Hochschule Freiburg)

(DD https://orcid.org/0000-0002-0812-461X

Hans-Werner Huneke

(Pädagogische Hochschule Heidelberg)

(D) https://orcid.org/0000-0001-5152-0207

\title{
Mehrsprachigkeit in Deutschland: Potenziale und Förderung im Herkunftssprachenunterricht
}

\section{Sprachenvielfalt in Deutschland: Mehrsprachigkeit und Migration}

„Mehrere Sprachen zu können ist eine Aufgabe und eine Leistung, an sich aber kein Problem. Zum Problem wird Menschen ihre Mehrsprachigkeit durch eine einsprachige Umgebung gemacht: eine Gesellschaft, die auf das Funktionieren in nur einer Sprache fixiert ist, und eine öffentliche Meinung, die durch die Sichtweise von Personen geprägt ist, die einsprachig aufgewachsen sind - zumindest ihrem Verständnis nach." Obwohl diese Zeilen in einem Aufsatz von Wilfried STöLting vor fast 15 Jahren verfasst worden sind (2005: 235), spiegeln sie zum Teil das nach wie vor kontrovers diskutierte Thema der migrationsbedingten Mehrsprachigkeit und Deutsch als Zweitsprache in der Bundesrepublik Deutschland wider. Auf der einen Seite wird die Mehrsprachigkeit als individuelle und gesellschaftliche Ressource angesehen, die in vielen (Lern-)Kontexten gewinnbringend eingesetzt werden kann, auf der anderen Seite wird primär die Förderung der Sprachkompetenzen in der Zielsprache Deutsch angestrebt. Damit rückt der Ausbau der Herkunftssprachen zu funktionalen Varietäten und damit die Förderung der 
Mehrsprachigkeit zunächst in den Hintergrund. Die Folge ist, dass die migrationsbedingte, lebensweltliche Mehrsprachigkeit von der Gesellschaft kaum wahrgenommen wird und sich nicht entfaltet (vgl. RöscH 2015).

Im folgenden Beitrag sollen zunächst einige Facetten und Formen der Mehrsprachigkeit und der mehrsprachigen Realität in Deutschland skizzenhaft vor allem in der Bildungsinstitution Schule vorgestellt werden. Anschließend soll aufgezeigt werden, welcher Stellenwert der Erziehung zur Mehrsprachigkeit im schulischen Kontext beigemessen wird. Wie die Mehrsprachigkeit gefördert werden kann, soll am Beispiel des Herkunftssprachenunterrichts Polnisch und Russisch dargestellt und diskutiert werden.

In der Mehrsprachigkeitsforschung wird zwischen unterschiedlichen Formen von Mehrsprachigkeit differenziert. So nimmt Riehl eine Einteilung in gesellschaftliche, institutionelle und individuelle Mehrsprachigkeit vor und definiert Mehrsprachigkeit als „verschiedene Formen von gesellschaftlich oder institutionell bedingtem und individuellem Gebrauch von mehr als einer Sprache“ (RIEHL 2014: 9). Gesellschaftliche Mehrsprachigkeit liegt dann vor, „wenn in einer Gesellschaft Sprecher verschiedener Sprachen faktisch darauf angewiesen sind und auch in der Lage sind, im Alltag miteinander zu kommunizieren und dies praktizieren, wenn also im Alltag mehrsprachige Konstellationen gegeben sind und die Zwecke, die darin durch sprachliches Handeln verfolgt werden, mittels verschiedener Typen ein- oder mehrsprachiger Diskurse [...] bearbeitet werden müssen [...]“ (Kameyama / ÖzDIL 2017: 71f.). Diese mehrsprachigen Konstellationen können in mehrsprachigen Staaten oder Regionen und städtischen Immigrantengruppen stattfinden (RIEHL 2014: 12). Institutionelle Mehrsprachigkeit ist dann gegeben, wenn staatliche Einrichtungen per Gesetz den Gebrauch mehrerer Sprachen zulassen und somit eine Verständigung in den anerkannten Sprachen ermöglicht wird (GARIBOva 2018: 32). Wenn von individueller Mehrsprachigkeit gesprochen wird, geht man vom Individuum aus, das über ein Sprachenrepertoire verfügt und sich je nach Gebrauchskontexten in der Interaktion für eine bestimmte Sprache entscheidet (WuLFF i.E.). Das Entscheidende dabei ist, dass die vorhandenen mehrsprachigen Ressourcen im Alltag genutzt werden und keine perfekte, einwandfreie Beherrschung der zwei oder mehreren Sprachen mündlich und schriftlich vorausgesetzt wird (KAMEYAMA / ÖZDIL 2017: 71).

Die Formen der Mehrsprachigkeit können noch um die innere Mehrsprachigkeit nach Mario Wandruszka (1981) mit Bezug auf Coseriu ergänzt werden: diatopische, diastratische und diaphasische Varietäten innerhalb einer Sprache. Daneben ist noch die fremdsprachliche Mehr- 
sprachigkeit zu benennen, die sich auf den schulischen Fremdsprachenunterricht bezieht.

Die Koexistenz mehrerer Sprachen gehört also zur gesellschaftlichen Realität in Deutschland, entgegen dem gesellschaftlichen Selbstverständnis von Einsprachigkeit, welches Gogolin (1994) als ,monolingualen Habitus' bezeichnet. Neben anerkannten Minderheitensprachen autochthoner Volksgruppen, Dänisch, Nord- und Saterfriesisch, Ober- und Niedersorbisch und Romani sowie der Regionalsprache Niederdeutsch, die alle durch die 1992 verabschiedete "Charta der Regional- und Minderheitensprachen" des Europarates geschützt werden, gibt es eine hohe Anzahl an Sprachen, die weder unter staatlichem Schutz stehen noch in der Gesellschaft auf Anerkennung stoßen. Die autochthone Minderheitensprachen-Vielfalt wird also ergänzt durch die migrationsbedingten Herkunftssprachen. Wenn man früher mit der Drei-GenerationenRegel rechnen konnte - die erste Generation beherrscht die Sprache des Ziellandes nur unvollständig, die zweite Generation ist zweisprachig, die dritte Generation verliert die Herkunftssprache (vgl. RiEHL 2014: 64) - so ist heutzutage von einer wesentlich größeren Vitalität der Herkunftssprachen auszugehen. Die mitgebrachten Sprachen werden durch Medien, technische Kommunikationsmittel und persönliche Mobilität unterstützt (GogOLIN 2005). Diese individuelle Mehrsprachigkeit ist jedoch nicht stabil, sondern dynamisch und Veränderungen unterworfen (Gogolin 2010).

Die Frage nach der Anzahl der mehrsprachigen Personen in Deutschland, ihren Sprachen und dem Kompetenzniveau in den jeweiligen Sprachen lässt sich nicht beantworten, da diese Angaben in amtlichen Statistiken nicht erfasst werden. Hier wird mit dem Hilfskonstrukt „Migrationshintergrund“ gearbeitet, das in Umfragen ermittelt wird. $\mathrm{Zu}$ Personen mit Migrationshintergrund werden Menschen gezählt, wenn sie selbst oder mindestens ein Elternteil nicht mit deutscher Staatsangehörigkeit geboren sind. 2018 hatten laut STATISTISCHem Bundesamt (2019) 20,78 Millionen Menschen, ca. 25 Prozent der Gesamtbevölkerung Deutschlands, einen Migrationshintergrund. Bei Kindern in der Altersgruppe 0 bis 5 Jahren ist mit rund 40 Prozent der höchste Anteil an Bevölkerung mit Migrationshintergrund zu verzeichnen. Diese Zahlen geben jedoch keine zuverlässige Auskunft darüber, ob die gezählten Personen mit Migrationshintergrund mehrsprachig sind. Über die Angaben zur Kategorie „Herkunftsland“ können die Zuzugsländer der zugewanderten Bevölkerung bestimmt werden. Jedoch sagen wiederum die auf diesem Weg ermittelten Daten noch nichts über die Erstsprachen bzw. die in den Familien verwendeten Sprachen der Personen mit Migrationshintergrund aus. Denn nicht alle, die in einem anderen Land zur 
Welt gekommen sind oder deren Eltern nach Deutschland zugewandert sind, sprechen automatisch eine der Amtssprachen des Herkunftslandes. Nichtsdestotrotz wird vom Herkunftsland der Personen mit Migrationshintergrund ausgehend versucht, auf deren Sprachen zu schließen.

Viel differenzierter und sensibler gehen die Studien mit den Herkunftssprachen um, die versuchen, die gelebte Sprachenvielfalt an Schulen zu ermitteln. So legen die Ergebnisse der Befragungen an Grundschulen in Hamburg, Essen und Freiburg (Fürstenau / Gogolin / Yagmur 2003, Chlosta / Ostermann 2010, Decker / Schnitzer 2013) nahe, dass bis zu 40 Prozent aller Grundschulkinder in der eigenen Familie noch eine weitere Sprache neben dem Deutschen verwenden. Insgesamt wurden bis zu 100 verschiedene Sprachen ermittelt, die die Schülerinnen und Schüler zusätzlich zum Deutschen sprechen. In der Essener Studie (Chlosta / Ostermann 2010) stand dabei das Türkische als eine besonders häufig genannte Herkunftssprache an der ersten Stelle, gefolgt vom Arabischen, Polnischen, Bosnischen / Kroatischen / Serbischen und Russischen.

Wenn man die Gesamtzahl der Polnisch- und Russischsprechenden zu ermitteln versucht, stößt man auf das oben dargelegte Problem. Man findet über Statistiken Informationen (STATISTISChES BundeSAmT 2019: 128), dass der Anteil der Bevölkerung Deutschlands mit polnischen Wurzeln im Jahr 2018 bei 2,25 Millionen, also bei rund 2,6 Prozent der Gesamtbevölkerung, lag. Dies sichert den Zugewanderten aus Polen den zweiten Platz in der Statistik nach der größten Zuwanderergruppe aus der Türkei. 1,7 Prozent der deutschen Population, rund 1,37 Millionen Menschen, sind aus der Russischen Föderation zugewandert. Bei diesen Angaben können aber beispielsweise die Personen nicht berücksichtigt werden, die aus den anderen Nachfolgestaaten der Sowjetunion in die Bundesrepublik zugewandert sind und deren Erstsprache Russisch ist. Die tatsächliche Anzahl russischsprachiger Bevölkerung Deutschlands kann deutlich höher als 1,37 Millionen eingeschätzt werden. Einigen Berechnungen zufolge (BREHMER 2007: 166) wird Russisch von mehr als drei Millionen Menschen gesprochen. Andere Wissenschaftler gehen von bis zu sechs Millionen Russischsprechenden aus (YILDIZ / GaGarina 2017: 43). Somit übersteigt die geschätzte Anzahl der Russischsprecher die statistisch erfassten Personen mit Wurzeln in der Russischen Föderation. Es ist anzunehmen, dass auch die Anzahl der Polnischsprechenden über der Anzahl der statistisch erfassten Personen mit polnischen Wurzeln liegt. Damit kann geschätzt werden, dass Polnisch und Russisch - neben Türkisch - zu den am häufigsten in Deutschland vertretenen Herkunftssprachen gehören (BURKHARDT ET AL. 2018: 165). 


\section{Förderung migrationsbedingter Mehrsprachigkeit}

In der Mehrsprachigkeitspolitik der EU wird sprachliche Diversität als wünschenswert angesehen: Jede europäische Bürgerin und jeder europäische Bürger sollte mindestens drei Sprachen beherrschen (KOM 2008/566). Dies führt u.a. dazu, dass in der Bildungsinstitution Schule zwei Fremdsprachen gelehrt und gelernt werden, bilingualer Sachfachunterricht wird an vielen Schulen praktiziert, auch frühes Fremdsprachenlernen gehört in vielen Kitas zum Alltag. Hierbei handelt es sich jedoch meistens um „klassische“ schulische Fremdsprachen wie Englisch, Französisch oder Spanisch. In Bezug auf die Förderung der migrationsbedingten Mehrsprachigkeit setzt Deutschland aufgrund der föderalen Verhältnisse keine einheitlichen Maßnahmen um. Stattdessen wird auf ein Mehrebenensystem zurückgegriffen, bei dem zwischen den Erlassen und Empfehlungen auf der Bundes-, Landes- und der Einzelschulebene unterschieden werden muss. In den Erlassen und Empfehlungen ist die Bestrebung festzustellen, „den monolingualen Habitus“ zu überwinden und die lebensweltliche herkunftsbedingte Mehrsprachigkeit der Schülerinnen und Schüler wahrzunehmen.

Auf der Bundesebene nimmt die ,Ständige Konferenz der Kultusminister der Länder“ (KMK) eine koordinierende Position ein und spricht Empfehlungen aus. In der Empfehlung „Interkulturelle Bildung und Erziehung in der Schule“ vom 5.12.2013 ist folgender Grundsatz enthalten: „Schule nimmt Vielfalt zugleich als Normalität und als Potenzial für alle wahr [Hervorhebung im Original]. Das bedeutet: [...] - Sie nimmt die sprachlich-kulturelle Vielfalt ihrer Schüler- und Elternschaft als Chance für interkulturelles Lernen bewusst wahr und berücksichtigt diese in der schulprogrammatischen Arbeit. Hierzu gehört auch die Würdigung und Förderung der sprachlichen Kompetenzen mehrsprachig aufwachsender Schülerinnen und Schüler“ (KMK 2013: 3). Dies soll unter anderem durch die „Wertschätzung und Anerkennung der herkunftsbedingten Mehrsprachigkeit“ geschehen (KMK 2013: 7). Ein weiterer Grundsatz der Empfehlung zur interkulturellen Bildung und Erziehung in der Schule lautet: „Schule ist ein zentraler Ort für den Erwerb bildungssprachlicher Kompetenzen“ [Hervorhebung im Original] (KMK 2013: 5). Dieser Grundsatz soll u.a. durch folgende Maßnahmen erreicht werden:

- Unterstützungsmaßnahmen für Schülerinnen und Schüler mit besonderem Sprachförderbedarf und Beachtung und Wertschätzung der Spracherwerbsprozesse von Zweitsprachlernenden,

- Nutzung der mehrsprachigen Kompetenzen von Schülerinnen und Schülern im Unterricht; Förderung mehrsprachiger Kompetenzen 
durch Unterrichtsangebote in den Herkunftssprachen der Schülerinnen und Schüler (KMK 2013: 8f.).

Auf der Landesebene wird die Umsetzung der Empfehlungen zur Förderung migrationsbedingterer Mehrsprachigkeit unterschiedlich gehandhabt. Ob und inwieweit die Empfehlungen angenommen werden, kann u.a. anhand von Lehrplänen und dem (Nicht-) Angebot vom herkunftssprachlichen Unterricht (HSU) festgestellt werden. So macht beispielsweise der LeHrPLAN Des LANDES NORDRHEIN-WestFalen (NRW) FÜR DIE GRUNDSChUle FÜR DAS FACH Deutsch (2008: 24) auf die Bedeutung sprachvergleichender Betrachtungen zwischen dem Deutschen und den Familiensprachen der Kinder aufmerksam. Diese sollen zur Erleichterung des Verständnisses möglicher Fehler der Schülerinnen und Schüler beitragen und die Kooperation mit den Lehrkräften für den Unterricht in der Herkunftssprache anregen. Auch die Kernlehrpläne weiterer Schulformen des Landes NRW für das Fach Deutsch berücksichtigen die mehrsprachigen Hintergründe der Lernenden als eine Ressource für die anzustrebenden Kompetenzerwartungen. Der KeRnleHrPlan FÜr DIE Realschule (2004: 19) berücksichtigt die Reflexion über Mehrsprachigkeit, die zur Entwicklung der Sprachbewusstheit und zum Sprachvergleich beiträgt. Ein Auszug aus dem KerNLEHRPLAN FÜr DAS GYMNASIUM DER SEKUNDARSTUFe I (2007: 12) lautet: „Kinder und Jugendliche anderer Herkunftssprachen können aus ihren Erfahrungen der Mehrsprachigkeit einen Beitrag zur vertieften Sprachkompetenz und Sprachbewusstheit leisten“. Auch hier werden also die migrationsbedingt mehrsprachigen Kompetenzen von Lernenden als Ressource verstanden.

\section{Herkunftssprachlicher Unterricht als Weg zur Mehrsprachigkeitsförderung}

Im Folgenden soll auf das Angebot des herkunftssprachlichen Unterrichts (HSU) als ein möglicher Weg für die Förderung der Mehrsprachigkeit näher eingegangen werden. Dazu erfolgt zunächst eine Definition des herkunftssprachlichen Unterrichts, bevor seine existierenden Angebotsformen in verschiedenen Bundesländern mit Lernerzahlen in den meistverbreiteten Herkunftssprachen Russisch und Polnisch sowie seine Rahmenbedingungen vorgestellt werden. Abschließend soll ein Blick auf die Erteilung dieser beiden Sprachen als Fremdsprachen und die Feststellung über die Eignung des Fremdsprachenunterrichts als ein Element der Förderung der Herkunftssprachen erfolgen. 
Bei dem herkunftssprachlichen Unterricht handelt es sich um einen besonderen Sprachunterricht, der die in der Familie erworbenen Sprachfähigkeiten von Sprechenden einer Herkunftssprache weiterentwickelt. Er unterscheidet sich vom Fremdsprachenunterricht und vom muttersprachlichen Unterricht im Herkunftsland.

Der herkunftssprachliche Unterricht setzt an der sprachlichen Primärsozialisation der Schülerinnen und Schüler an. Er nutzt die in der Familienkommunikation erworbenen Fähigkeiten, die durch peers-Kontakte, Vereinsaktivitäten, Mediennutzung und temporäre Aufenthalte in einem Gebiet der Herkunftssprache verstärkt sein können, um diese auszubauen und weiterzuführen. Er ist kein Fremdsprachenunterricht, der bei null beginnt und dann systematisch voranschreitet, sondern ein Unterricht auf der Basis außerschulischer Kommunikation. Zugleich hat er sich jedoch darauf einzustellen, dass es sich dabei um Kommunikation in einer Minderheitensprache handelt, die im Einwanderungsland nur für einen begrenzten Teil der gesellschaftlichen Anforderungen Verwendung findet. Er kann darum auch kein Abbild des muttersprachlichen Unterrichts sein, wie er in den Schulen des Herkunftslandes erteilt wird. (ReIch 2018: 294)

Die institutionelle Verankerung des herkunftssprachlichen Unterrichts geht auf eine Vielzahl von auf der europäischen und nationalen Ebene verabschiedeten Beschlüssen und Erlassen zurück, die schulische Maßnahmen schulpflichtiger ausländischer Nachkommen von damals als „Gastarbeiter“ Bezeichneten betreffen. Auf der Bundesebene hat die Kultusministerkonferenz 1971 beschlossen, dass jedes Bundesland in eigener Zuständigkeit darüber entscheidet, „ob dieser Unterricht innerhalb oder außerhalb des Verantwortungsbereichs der Kultusverwaltung steht“ (zitiert nach ReIch 2014: 2). Die Bundesländer haben die Entscheidung über die Verantwortung des HSU also nicht einheitlich getroffen. Heute hat sich die Zusammensetzung der Schülerschaft im HSU wesentlich geändert, aber die Uneinheitlichkeit in den Rahmenbedingungen besteht weiter. Es existieren unterschiedliche Formen des HSU, die zum Teil parallel realisiert werden.

In vier Bundesländern (Mecklenburg-Vorpommern, Brandenburg, Sachsen, Rheinland-Pfalz) steht der HSU ausschließlich unter Verantwortung des jeweiligen Bundeslandes, welches sich um die Organisation des Unterrichts und die Einstellung von Lehrkräften kümmert. In Schleswig-Holstein, Bayern und Baden-Württemberg steht HSU unter Aufsicht von diplomatischen Vertretungen. In den meisten Bundesländern (Berlin, Hamburg, Bremen, Nordrhein-Westfalen, Niedersachsen, Hessen, Saarland) besteht die Möglichkeit, entweder am von den Schul- 
aufsichten staatlich angebotenen oder von den Konsulaten durchgeführten Unterricht teilzunehmen. Es existieren also zwei parallele Angebote. In Thüringen und Sachsen-Anhalt wird herkunftssprachlicher Unterricht weder von diplomatischen Vertretungen noch von staatlichen Schuleinrichtungen durchgeführt (vgl. Mediendienst InTEgration 2019).

Die folgende Abbildung 1 illustriert, in welcher Form herkunftssprachlicher Unterricht im Schuljahr 2018/2019 in der Bundesrepublik Deutschland erteilt wurde. In der Abbildung sind auch Angaben zu Teilnehmenden des Herkunftssprachenunterrichts in Polnisch und Russisch für das Schuljahr 2018/2019 enthalten, die dem Bericht vom Mediendienst Integration entnommen wurden. Der Bericht wurde auf Grundlage einer Anfrage bei der Kultusministerkonferenz und der übermittelten Daten zum Herkunftssprachenunterricht der Länder erstellt.

In den Bundesländern, in denen keine Zahlen zu Lernenden eingetragen sind, wurde der Unterricht in Herkunftssprachen Polnisch und Russisch unter staatlicher oder diplomatischer Verantwortung entweder nicht erteilt oder es liegen keine Angaben hierzu vor. Zahlen zum Besuch privater Angebote zum Herkunftssprachenunterricht, die zum Beispiel von Vereinen oder Elterninitiativen veranstaltet wurden, wurden nicht erfasst und sind in der Übersicht nicht enthalten.

Das Land Baden-Württemberg bietet derzeit den Konsulatsunterricht in 14 Sprachen an, u.a. auch in Polnisch. Im Schuljahr 2018/2019 nahmen in Baden-Württemberg 230 Schülerinnen und Schüler am Konsulatsunterricht in Polnisch als Herkunftssprache teil. In Brandenburg besuchten 224 Schülerinnen und Schüler den staatlichen Unterricht in der Herkunftssprache Polnisch und 214 Schülerinnen und Schüler in Russisch. In Hamburg gab es 12 Lerngruppen, die Russisch als Herkunftssprache unter staatlicher Verantwortung lernten, und 8 Lerngruppen für Polnisch als Herkunftssprache. Hessen bot in eigener Zuständigkeit Polnischunterricht an, an dem 479 Lernende teilnahmen. In MecklenburgVorpommern nutzten den Unterricht in Polnisch als Herkunftssprache 340 Lernende. Die meisten Lernenden des Russischen und Polnischen als Herkunftssprache, die den landeseigenen Unterricht besuchten, gab es den Statistiken zufolge in Nordrhein-Westfalen. Dort nahmen 10.777 Lernende am Russischunterricht als Herkunftssprache teil, Polnischunterricht als Herkunftssprache besuchten 4.636 Schülerinnen und Schüler. In Rheinland-Pfalz machten vom staatlichen Angebot in Russisch als Herkunftssprache 2.361 Lernende und in Polnisch als Herkunftssprache 583 Lernende Gebrauch. In Sachsen besuchten 462 Schülerinnen und Schüler den Unterricht in Russisch als Herkunftssprache und 191 Schülerinnen und Schüler den Polnischunterricht als Herkunftssprache. In Niedersachsen lagen nur Zahlen für das Schuljahr 2017/2018 vor. Aus 
SU Polnisch: 8 Lerngruppen

SU Russisch: 12 Lerngruppen
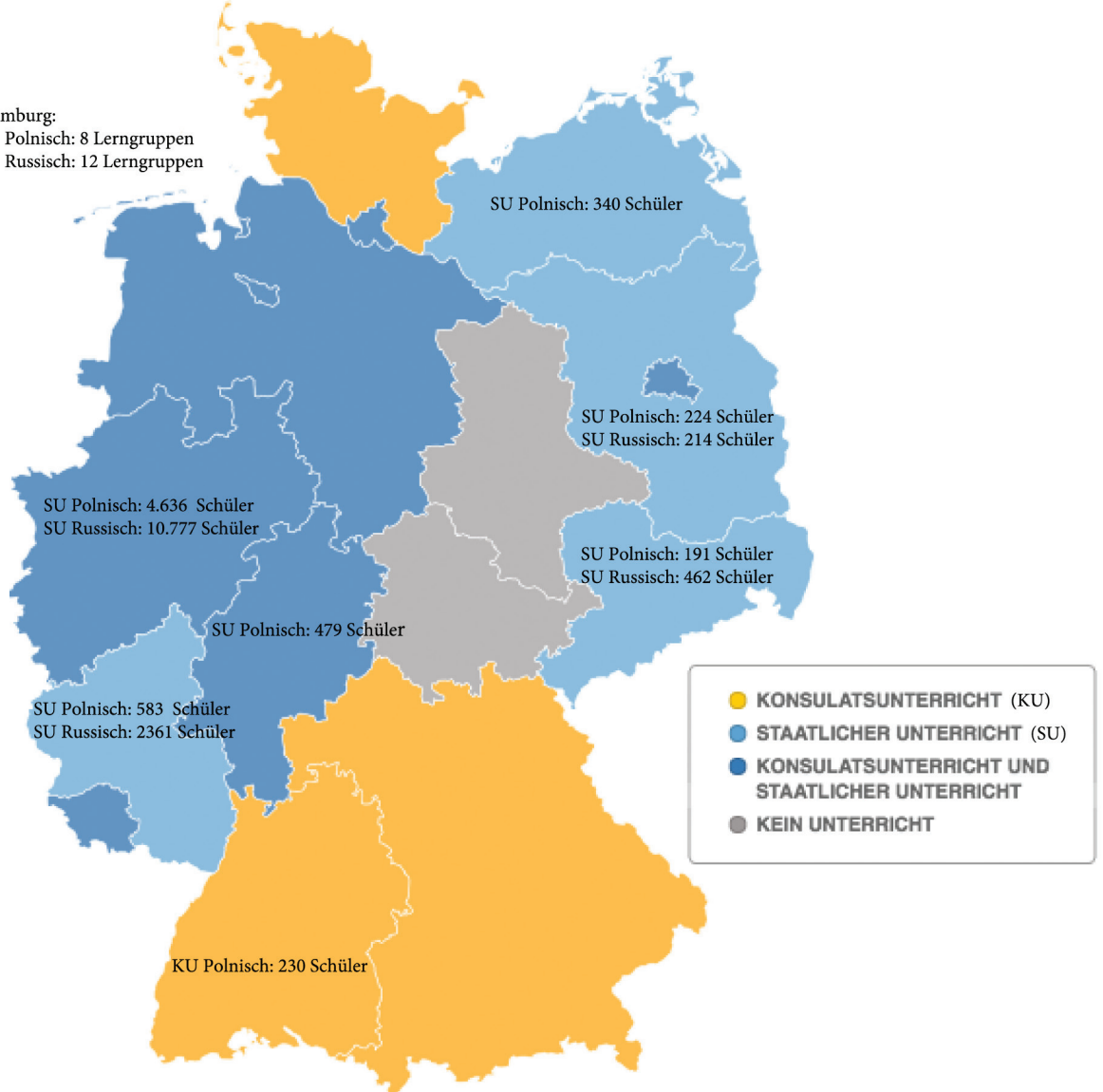

Abb. 1: Herkunftssprachlicher Unterricht in Deutschland 2018/2019 und Lernerzahlen im Schuljahr 2018/2019 für den Herkunftssprachenunterricht in Polnisch und Russisch. (Quelle: Mediendienst Integration, 2019: 4)

diesem Grund sind sie der Abbildung 1 nicht zu entnehmen. Damals nahmen 755 Kinder und Jugendliche am Unterricht in Russisch und 398 in Polnisch als Herkunftssprache teil.

Die Angaben zu den Besuchszahlen können nicht als vollständig und genau gelten, denn wie Lengyel in ihrer Stellungnahme zur Abfrage des Mediendienstes Integration zur Situation des Herkunftssprachenunterrichts in den Bundesländern feststellt, ist

[d]ie vorliegende Datenlage [...] desolat, denn die von den Ländern geführten Statistiken sind häufig lückenhaft: So gibt es Länder, in denen Statistiken zu den teilnehmenden Schülerinnen und Schülern überhaupt nicht geführt werden und die anscheinend erst anlassbezo- 
gen (etwa durch Abfragen wie die vorliegende) Zahlen einholen. Auch dies kann als Ausdruck des geringen Stellenwerts angesehen werden, der dem HU [=Herkunftssprachenunterricht, Anm. der Autoren] beigemessen wird. Daher kann auf dieser Basis auch kaum eine verlässliche Einschätzung zu den Besuchszahlen im Zeitverlauf getroffen werden (LENGYeL 2019: 2f.).

Die Rahmenbedingungen des herkunftssprachlichen Unterrichts und die Leistungen, die darin erbracht werden können, sind als heterogen zu charakterisieren. Je nach Organisationsform wird der HSU unterschiedlich anerkannt. Er kann anstelle einer Fremdsprache treten und versetzungsrelevant sein. Oft wird er jedoch als zusätzlicher, jahrgangsübergreifender Unterricht erteilt, was dazu führt, dass die Teilnehmenden unterschiedlich alt sind. Auch die sprachlichen Fertigkeiten der Herkunftssprachensprechenden können stark variieren. Der Unterricht richtet sich sowohl an rezeptiv Bilinguale, die lediglich die Herkunftssprache verstehen, jedoch nicht sprechen können, als auch an Schülerinnen und Schüler, die ausgeprägte literale Kompetenzen in der Herkunftssprache besitzen. Für das Stundenvolumen existieren keine einheitlichen Standards. Es werden ca. zwischen zwei und fünf Wochenstunden Unterricht in der Herkunftssprache erteilt. Der Unterricht in Herkunftssprachen wird am häufigsten von Kindern der Primarstufe besucht. Die Teilnahme am HSU ist nicht obligatorisch und kann jederzeit bzw. - bei staatlich organisiertem Unterricht - zum Ende des Schul(halb)jahres beendet werden (BREhmer/MehlHorn 2018: 71f.).

Es lässt sich feststellen, dass es aufgrund der föderalen Bildungspolitik und der Unsicherheit über die Legitimation des herkunftssprachlichen Unterrichts Unübersichtlichkeiten und Widersprüchlichkeiten rund um diesen Unterricht gibt, die zu seiner randständigen Position im deutschen Bildungssystem führen (REICH 2014: 3). Obwohl Herkunftssprachenunterricht einen Beitrag zum Ausbau der Mehrsprachigkeitsbildung leisten kann, ist er in der Regel nicht mit weiteren schulischen Angeboten zur sprachlichen Bildung verzahnt. Auch auf das mehrsprachige Potenzial der Lernenden im Herkunftssprachenunterricht wird kaum eingegangen. Dadurch gehen wertvolle Ressourcen verloren.

Es existieren Konzepte zur Verzahnung zwischen dem HSU und dem Regelunterricht. Einige wenige Grundschulen in NRW bieten die Möglichkeit einer bilingualen koordinierten Alphabetisierung (KOALA) in Herkunftssprachen an. In enger Abstimmung zwischen den Deutschund HSU-Lehrenden erfolgen die Einführung des Alphabets und die Vermittlung von Lese- und Schreibfertigkeiten. Zusätzlich zur zweisprachigen Alphabetisierung wird die jeweilige Partnersprache im Sach- und 
Sprachunterricht über die gesamte Grundschulzeit mitberücksichtigt, wodurch der Ausbau schrift- und bildungssprachlicher Kompetenzen in zwei Sprachen ermöglicht wird. Ein Teil der Unterrichtseinheiten erfolgt dabei im Team-Teaching (BezIrKSREgIERUNG KöLN 2014).

Bei der Durchführung der Evaluation zum KOALA-Konzept wurden drei Gruppen miteinander verglichen: eine Gruppe, die nach dem KOALA-Konzept unterrichtet wurde, eine weitere Gruppe, die Deutschfördermaßnahmen erhielt, eine andere Gruppe, die neben regulärem Deutschunterricht am Herkunftssprachenunterricht teilnahm. Die Evaluationsergebnisse haben gezeigt, dass die Gruppe, die nach dem KOALA-Konzept unterrichtet wurde, die besten Ergebnisse bei der Textproduktion in beiden Sprachen erreichte. Die Gruppe, die neben dem regulären Deutschunterricht am Herkunftssprachenunterricht teilnahm, erzielte die schlechtesten Ergebnisse in der Untersuchung (ReIcH 2011: 15f.). Dadurch kann das Fazit gezogen werden, dass die Förderung der gesamten sprachlichen Kompetenzen in strukturierten zweisprachigen Modellen besonders erfolgversprechend ist. Wird Herkunftssprachenunterricht nur additiv erteilt und die Herkunftssprache im weiteren Lernprozess nicht mitberücksichtigt, geht der Lernerfolg verloren.

\section{Herkunftssprachen als Fremdsprachen}

Manche Herkunftssprachen, darunter Polnisch und Russisch, können auch als Fremdsprachen regulär an Schulen erlernt werden.

In allen 16 Ländern der Bundesrepublik Deutschlands besteht die Möglichkeit, Russischunterricht an Schulen zu erhalten und - bis auf das Saarland - als Abiturfach zu belegen. Russischunterricht bieten jedoch bei weitem nicht alle Schulen an. Die Möglichkeiten dafür sind regional sehr unterschiedlich. Nach den Lernerzahlen belegt Russisch im Schuljahr 2017/2018 mit rund 106.000 Fremdsprachenlernern nach Englisch, Französisch, Latein und Spanisch den fünften Platz der am häufigsten gelernten Fremdsprachen an allgemeinbildenden Schulen. An beruflichen Schulen lernen im Schuljahr 2017/2018 11.447 Schülerinnen und Schüler Russisch als Fremdsprache (Statistisches Bundesamt 2018). Auch Polnisch wird vereinzelt als Fremdsprache an Schulen angeboten, allerding fehlen hierzu verlässliche Zahlen.

Herkunftssprechende können von ihren Vorkenntnissen im Fremdsprachenunterricht profitieren. Dennoch stellt diese Gruppe von Lernenden die Lehrkräfte im schulischen Fremdsprachenunterricht häufig vor didaktisch-methodische Herausforderungen, Schülerinnen und Schüler mit und ohne Vorkenntnisse gemeinsam zu unterrichten, denn 
in Lehrwerken und Lehrplänen wird die zweisprachige Lebenswelt von Sprechenden der Herkunftssprachen kaum berücksichtigt. Diese Umstände können bei Schülerinnen und Schülern, die die zu erlernende Fremdsprache bereits als Herkunftssprache sprechen, Unterforderung und Frustration verursachen. Es besteht ein Bedarf nach geeigneten Lehrkonzepten und nach Binnendifferenzierung im Fremdsprachenunterricht sowie nach der Entwicklung einer inklusiven Didaktik, die die Bedürfnisse aller Lernenden abdeckt (Brehmer / Mehlhorn / YastreBOVA 2017: 693 ff.).

\section{Fazit}

Das deutsche Schulsystem steht vor der Herausforderung, die migrationsbedingte Mehrsprachigkeit seiner Lernenden anzuerkennen, diese aus ihrer Randständigkeit herauszuholen und sie in sinnvolle Sprachlernkonzepte sowie in den Fachunterricht zu integrieren. Fremdsprachenunterricht in allen Herkunftssprachen anzubieten, scheint weder umsetzbar zu sein noch aus der Sicht der Mehrsprachigkeitsforschung eine erfolgsversprechende Wirkung zu haben. Eine mehrsprachige und sprachsensibel arbeitende Schule bedarf daher der Entwicklung und Umsetzung fächerübergreifender Sprachlernkonzepte, die die Mehrsprachigkeit der Schülerinnen und Schüler als Ressource verstehen und den Einbezug der Herkunftssprachen im Unterricht ermöglichen. Wenn es Aufgabe der Schule ist, alle Kinder bestmöglich nach ihren Voraussetzungen, Begabungen und Möglichkeiten zu fördern, sollten sie auch in ihrer Mehrsprachigkeit gefördert werden.

\section{Literatur}

BeZirksRegierung KöLn (2014): Koordinierte Alphabetisierung im Anfangsunterricht. Das KOALA-Konzept an Kölner Schulen. Unveröffentlichtes Manuskript. In: https://www.bezreg-koeln.nrw.de/brk_internet/publikationen/ abteilung04/pub_abteilung_04_koala.pdf (23.09.2020)

Brenmer Bernhard (2007): Sprechen Sie Qwelja? Formen und folgen russischdeutscher Zweisprachigkeit in Deutschland. In: AnstaTt Tanja (Hg.): Mehrsprachigkeit bei Kindern und Erwachsenen. Tübingen: Attempto: 163-185.

Brehmer Bernhard / Mehlhorn Grit / Yastrebova Maria (2017): Die Herkunftssprache Russisch als Ressource im Sprachunterricht. In: WITZLACK-MAKAREvich Kai / WulfF Nadja (Hg.): Handbuch des Russischen in Deutschland: Migration - Mehrsprachigkeit - Spracherwerb. Berlin: Frank \& Timme: 687699. 
Katharina Hamann, Nadja Wulff, Hans-Werner Huneke: Mehrsprachigkeit...

Brehmer Bernhard / MehlHorn Grit (2018): Herkunftssprachen. Tübingen: Narr. Burkhardt Joanna / Mehlhorn Grit / Yastrebova Maria (2018): Spracheinstellungen in polnisch- und russischsprachigen Familien in Deutschland. In: Mehlhorn Grit / Brehmer Bernhard (Hg.): Potenziale von Herkunftssprachen. Tübingen: Stauffenburg: 1651-83.

Chlosta Christoph / Ostermann Torsten / Schroeder Christoph (2010): Grunddaten zur Mehrsprachigkeit im deutschen Bildungssystem. In: BERNT Ahrenholz / OOMEN-Welke Ingelore (Hg.): Deutsch als Zweisprache. DTP 9. Baltmannsweiler: Schneider Verlag Hohengehren: 17-30.

Decker Yvonne / Schnitzer Katja (2013): Sprachförderung von Kindern nicht deutscher Herkunftssprache in Vorbereitungsklassen Baden-Württembergs. Eine explorative Studie. In: Whadimir Kosov / Huneke Hans-Werner (Hg.): Aktuelle Forschungsbeiträge aus bildungswissenschaftlichen Universitäten. Freiburg: Verlag der Pädagogischen Hochschule: 1441-55.

Fürstenau Sara / Gogolin Ingrid / YaĞMUR Kutlay (Hg.) (2003): Mehrsprachigkeit in Hamburg. Ergebnisse einer Spracherhebung an den Grundschulen in Hamburg. Münster: Waxmann.

Garibova Jala (2018): Historische und kommunikative Aspekte. In: Roche Jörg / Terrasi-Haufe Elisabetta (Hg.): Mehrsprachigkeit und Spracherwerb. Tübingen: Narr Francke Attempo: 293-9.

Gogolin Ingrid (1994): Der monolinguale Habitus der multilingualen Schule. Münster. Waxmann.

Gogolin Ingrid (2005): Erziehungsziel Mehrsprachigkeit. In: RöHnER Charlotte (Hg.): Erziehungsziel Mehrsprachigkeit. Diagnose von Sprachentwicklung und Förderung von Deutsch als Zweitsprache. Weinheim/München: Juventa Verlag: $13-24$.

GogolIN Ingrid (2010): Stichwort Mehrsprachigkeit. In: Zeitschrift für Erziehungswissenschaft, Heft 13: 529-547.

Kameyama Shinichi / Özdil Erkan (2017): Mehrsprachigkeit. In: HoffmanN Ludger / Kameyama Shinichi / Riedel Monika / Sahiner Pembe / WulfF Nadja (Hg.): Deutsch als Zweitsprache: Grundlagen für die Lehrerausbildung. Berlin: Erich Schmidt: 71-90.

KOM 2008/566 = Kommission DER EuropäIschen GemeInschaften: Mitteilung der Kommission an das Europäische Parlament, den Rat, den Europäischen Wirtschafts- und Sozialausschuss und den Ausschuss der Regionen - Mehrsprachigkeit: Trumpfkarte Europas, aber auch gemeinsame Verpflichtung. In: https://eur-lex.europa.eu/legal-content/DE/TXT/PDF/?uri=CELEX:52008D C0566\&from=DE (12.08.2019)

LeNGyel Drorit (2019): Stellungnahme zur Abfrage des Mediendienstes Integration zur Situation des Herkunftssprachenunterrichts (HU) in den Bundesländern.

MEDIENDIENST INTEGRATION (2019): Wie verbreitet ist herkunftssprachlicher Unterricht? In: https://mediendienst-integration.de/fileadmin/Herkunftssprachlicher_Unterricht_2019.pdf (23.09.2020). 
Ministerium FÜR SChule Und WeIterbildung Des LANDES IN NordrheinWestFalen (2004): Kernlehrplan für die Realschule in Nordrhein-Westfalen. Deutsch. Frechen: Ritterbach Verlag GmbH.

Ministerium für Schule Und Weiterbildung des Landes in Nordrhein-WestFALEN (2007): Kernlehrplan für den verkürzten Bildungsgang des Gymnasiums - Sekundarstufe I (G8) in Nordrhein-Westfalen. Deutsch. Frechen: Ritterbach Verlag GmbH.

Ministerium für SCHUle Und Weiterbildung des Landes in Nordrhein-WestFALEN (2008): Richtlinien und Lehrpläne für die Grundschule in NordrheinWestfalen. Deutsch, Sachunterricht, Mathematik, Englisch, Musik, Kunst, Sport, Evangelische Religionslehre, Katholische Religionslehre. Frechen: Ritterbach Verlag GmbH.

ReICH Hans-H. (2011): Schriftsprachliche Fähigkeiten türkisch-deutscher Grundschülerinnen und Grundschüler in Köln. In: https://www.bezreg-koeln.nrw. de/brk_internet/publikationen/abteilung04/pub_abteilung_04_reich_evalu ierung.pdf (23.09.2020).

ReICH Hans H. (2014): Über die Zukunft des Herkunftssprachlichen Unterrichts. Überarbeitete Fassung eines Vortrags bei der GEW Rheinland-Pfalz in Mainz am 31.01.2012. In: https://www.uni-due.de/imperia/md/content/prodaz/ reich_hsu_prodaz.pdf (23.09.2020).

ReICH Hans H. (2018): Herkunftssprachlicher Unterricht aus curricularer Sicht. In: Mehlhorn Grit / Brehmer Bernhard (Hg.): Potenziale von Herkunftssprachen - Sprachliche uns außersprachliche Einflussfaktoren. Tübingen: Stauffenburg: 295-298.

RIEHL Claudia Maria (2014): Mehrsprachigkeit: Eine Einführung. Darmstadt: Wissenschaftliche Buchgesellschaft.

Rösch Heidi (2015): Sprach(en)bewusstheit als Sprachbildungskonzept. In: DitTManN Alina / GiblaK Beata / WiTt Monika (Hg.): Bildungsziel: Mehrsprachigkeit - Towards the Aim of Education: Multilingualism. Leipzig: Leipziger Universitätsverlag: 93-108.

STÄNDIGE KONFERENZ DER KultuSMinister DER LÄNDER (1971): Empfehlung: Unterricht für Kinder ausländischer Arbeitnehmer. Beschluss der Kultusministerkonferenz vom 3.12.1971. Berlin: Sekretariat der Ständigen Konferenz der Kultusminister in der Bundesrepublik Deutschland.

STÄNDIGE KONFERENZ DER KultuSMINISTER DER LÄNDER (2013): Interkulturelle Bildung und Erziehung in der Schule. (Beschluss der Kultusministerkonferenz vom 25.10.1996 i. d. F. vom 05.12.2013.) Berlin: Sekretariat der Ständigen Konferenz der Kultusminister in der Bundesrepublik Deutschland. In: https://www.kmk.org/fileadmin/Dateien/.../1996_10_25-Interkulturelle-Bil dung.pdf (23.09.2020).

StATISTISCHES BundeSAMT (2018): Schüler/innen mit fremdsprachlichem Unterricht. Wiesbaden. In: https://www.destatis.de/DE/Themen/GesellschaftUmwelt/Bildung-Forschung-Kultur/Schulen/Tabellen/allgemeinbildendeberuflicheschulen-fremdsprachl-unterricht.html (23.09.2020). 
Katharina Hamann, Nadja Wulff, Hans-Werner Huneke: Mehrsprachigkeit...

WandruszKa Mario (1981): Die Mehrsprachigkeit des Menschen. München: DTV HäUSER Katrin / Haberzettl Stefanie / Heine Antje (Hg.): Handbuch Deutsch als Fremd- und Zweitsprache. Stuttgart: J.B. Metzler Verlag.

Yildiz Cemal; Gagarina Natalia (2017): Eröffnungsrede. In: Yildiz Cemal / ToPAJ Nathalie / Thomas Reyhan / Gülzow Inza (Hg.): Die Zukunft der Mehrsprachigkeit im deutschen Bildungssystem: Russisch und Türkisch im Fokus. Frankfurt am Main: Peter Lang: 39-43.

Zusammenfassung: Durch die anhaltende Einwanderung in den letzten Jahrzehnten hat sich die Bevölkerungszusammensetzung Deutschlands stark verändert. Das Land hat an neuen Bürgerinnen und Bürgern und damit auch an neuem sprachlichen Reichtum gewonnen. Welchen Stellenwert die neue migrationsbedingte Sprachenvielfalt hat und wie die mehrsprachigen Ressourcen vor allem im Kontext Schule erschlossen werden können bzw. sollten, wird intensiv diskutiert. Im Herkunftssprachenunterricht sollen die Erstsprachen der Schülerinnen und Schüler zu funktionalen Varietäten aufund ausgebaut werden. Im folgenden Beitrag soll dargestellt werden, wie die Mehrsprachigkeit im Rahmen des Herkunftssprachenunterrichts Russisch und Polnisch gefördert wird und welche Herausforderungen und Potenziale diese Unterrichtsform mit sich bringt.

Schlagwörter: Mehrsprachigkeit, Herkunftssprache, Russisch, Polnisch

Katharina Hamann studierte Deutsch als Fremd- und Zweitsprache an der Universität Bielefeld, Deutschland. Aktuell ist sie für den Bereich Deutsch als Zweitsprache in der Lehramtsausbildung der Universität Wuppertal zuständig. Ihre Forschungsinteressen sind Mehrsprachigkeit und Russisch als Herkunftssprache.

Dr. Nadja Wulff koordiniert als Juniorprofessorin an der Pädagogischen Hochschule Freiburg das Promotionskolleg „Vom fachsensiblen Sprachunterricht zum sprachsensiblen Fachunterricht“, das gemeinsame Projekt der sechs Pädagogischen Hochschulen in Baden-Württemberg. Zu ihren Forschungsschwerpunkten gehören Didaktik und Methodik des Deutschen als Zweit- und Fremdsprache, insbesondere Wortschatzvermittlung, sprachliches und fachliches Lernen sowie Sprachvergleich im Unterricht.

Prof. Dr. Hans-Werner Huneke studierte Deutsch und Geschichte an den Universitäten Hamburg und Münster. Er war als Lehrer in Brasilien und Deutschland tätig, als DAAD-Lektor an der Universität Coimbra/Portugal und an den Pädagogischen Hochschulen Heidelberg und Ludwigsburg. Nach der Promotion folgte er dem Ruf an die Pädagogische Hochschule Freiburg. Seit 2015 ist er Rektor der PH Heidelberg. Zu seinen 
Forschungsinteressen gehören Didaktik und Methodik des Deutschen als Erst- und Fremdsprache, Schriftspracherwerb und Schreibdidaktik.

\section{Wielojęzyczność w Niemczech: potencjał oraz możliwości jej rozwijania $i$ promowania na zajęciach $z$ języka ojczystego}

Streszczenie: Ze względu na utrzymującą się w ostatnich dziesięcioleciach imigrację skład ludności Niemiec znacząco się zmienił. Kraj ten zyskał nowych obywateli, a tym samym nowe bogactwo językowe, co prowadzi do intensywnych dyskusji nad znaczeniem nowej różnorodności językowej związanej z migracją oraz tego, jak można i należy rozwijać zasoby wielojęzyczne, szczególnie w kontekście szkolnym. Wskazuje się na to, aby na zajęciach z języka ojczystego pracować nad rozwijaniem języków ojczystych uczniów pod kątem różnorodności funkcjonalnej. Celem niniejszego artykułu jest ukazanie możliwości rozwijania i promowania wielojęzyczności w ramach nauczania języka rosyjskiego i polskiego oraz potencjału tego rodzaju nauczania, a także wyzwań przed nim stojących.

Słowa-klucze: wielojęzyczność, język ojczysty, rosyjski, polski

Katharina Hamann studiowała język niemiecki jako język obcy na Uniwersytecie w Bielefeld, Niemcy. Obecnie pracuje na Uniwersytecie w Wuppertalu i jest odpowiedzialna za kształcenie przyszłych nauczycieli w obszarze języka niemieckiego jako języka drugiego. Jej zainteresowania naukowo-badawcze to wielojęzyczność oraz język rosyjski jako język ojczysty.

Dr. Nadja Wulff jest adiunktem w Wyższej Szkole Pedagogicznej we Freiburgu i koordynuje wspólny projekt sześciu Wyższych Szkół Pedagogicznych w Baden-Württemberg: „Vom fachsensiblen Sprachunterricht zum sprachsensiblen Fachunterricht”. W polu jej zainteresowań badawczych znajdują się dydaktyka i metodyka nauczania języka niemieckiego jako języka drugiego i obcego ze szczególnym uwzględnieniem pracy ze słownictwem, uczenia się języka oraz przedmiotowego, jak również aspekty porównawcze.

Prof. Dr. Hans-Werner Huneke studiował język niemiecki oraz historię na Uniwersytetach w Hamburgu i Münster. Pracował jako nauczyciel w Brazylii i w Niemczech, a także jako lektor DAAD na uniwersytecie Combra w Portugalii oraz w Wyższej Szkole Pedagogicznej w Heidelbergu i Ludwigsburgu. Po uzyskaniu stopnia doktora został zatrudniony w Wyższej Szkole Pedagogicznej we Freiburgu. Od 2015 roku jest rektorem Wyższej Szkoły Pedagogicznej w Heidelbergu. Jego zainteresowania naukowo-badawcze oscylują wokół dydaktyki i metodyki nauczania języka niemieckiego jako języka pierwszego i obcego, a także dotyczą procesu nabywania i rozwijania umiejętności pisania i jego dydaktyki. 
Summary: Due to ongoing immigration in the last few decades, the composition of the population in Germany has changed significantly. The country has gained new citizens and with them came also new linguistic wealth. The importance of the new migration-related linguistic diversity and how the multilingual resources can or should be developed, especially in the school context, are topics of heated debate. In the native language lessons, the pupils' first languages should be developed and expanded into functional varieties. The aim of the present article is to show how multilingualism is promoted in the context of Russian and Polish language teaching, and what challenges and potentials this type of teaching brings with it.

Keywords: multilingualism, the native language, Russian, Polish

Katharina Hamann studied German as a foreign and second language at Bielefeld University, Germany. She is currently responsible for German as a second language in teacher training at the University of Wuppertal. Her research interests are multilingualism and Russian as the native language.

Nadja Wulff, PhD, as an assistant professor at the University of Education in Freiburg, she coordinates the joint project of the six teaching universities in Baden-Württemberg „From subject-sensitive language teaching to language-sensitive subject teaching”. Her research focuses on the didactics and methodology of German as a second and foreign language, in particular vocabulary teaching, language and subject learning, and language comparison in class.

Prof. Hans-Werner Huneke, PhD, studied German and history at the Universities of Hamburg and Münster. He worked as a teacher in Brazil and Germany, as a DAAD lecturer at the University of Coimbra (Portugal) and at the Heidelberg and Ludwigsburg Universities of Education. After completing his doctorate, he accepted a position at the Freiburg University of Education. He has been Rector of the University of Education in Heidelberg since 2015. His research interests include didactics and methodology of German as a first and foreign language, written language acquisition and teaching of writing. 